Review Article

\title{
The Safety and Effectiveness of Bevacizumab in the Treatment of Nonsquamous Non-Small-Cell Lung Cancer: A Meta-Analysis of Randomized Controlled Trials
}

\author{
Yue Zhou, Mei He, Rui Li, Yuan Peng, Feng Li, Shengqian Li, and Ming Yang $\mathbb{D}$ \\ Department of Pharmacy, Affiliated Hospital of North Sichuan Medical College, China \\ Correspondence should be addressed to Ming Yang; yangming1211@nsmc.edu.cn
}

Received 24 February 2021; Revised 27 July 2021; Accepted 12 August 2021; Published 7 September 2021

Academic Editor: Ugo Cioffi

Copyright (c) 2021 Yue Zhou et al. This is an open access article distributed under the Creative Commons Attribution License, which permits unrestricted use, distribution, and reproduction in any medium, provided the original work is properly cited.

\begin{abstract}
Objective. Bevacizumab was currently available for nonsquamous non-small-cell lung cancer (NSqNSCLC) patients and has been studied in several randomized controlled trials (RCTs) for treatment of these patients. This meta-analysis summarizes the most up-to-date evidences regarding the effects and adverse reactions of bevacizumab in the treatment of NSqNSCLC patients. Methods. The authors searched for RCTs from electronic database including PubMed, EMBASE, and the Cochrane Central Register of Controlled Trials. Experimental arm was defined as the bevacizumab-containing group and the control arm as the bevacizumab-free group. Data of objective response rate (ORR), disease control rate (DCR), progression-free survival (PFS), overall survival (OS), and adverse reactions were synthetically extracted. A protocol for this meta-analysis has been registered on PROSPERO (http://www.crd.york.ac.uk/prospero). Results. Ten RCTs that involved a total of 3134 patients were included. The experimental group was associated with significant superior ORR (RR 1.63, 95\% CI 1.24 to 2.14, $P<0.001$ ), OS (HR 0.90, 95\% CI 0.82 to $0.99, P<0.001$ ), and prolonged PFS (HR 0.68, 95\% CI 0.62 to $0.74, P<0.001$ ) compared to the control. No significant difference was observed regarding DCR (RR $1.13,95 \%$ CI 0.99 to $1.30, P=0.08$ ). The experimental group showed higher rate of hypertension (RR 6.91,95\% CI 4.62 to $10.35, P<0.00001$ ) and hemorrhagic events (RR 3.07, 95\% CI 1.78 to $5.30, P<0.0001$ ) than the control group. The experimental group showed lower rate of anemia (RR $0.72,95 \%$ CI 0.55 to 0.96 , $P=0.02)$ than the control group. No significant difference was observed regarding treatment-related adverse event grade 3-5 (TRAE3-5) (RR $1.23,95 \%$ CI 0.99 to $1.53, P=0.06$ ), thrombocytopenia (RR $1.11,95 \%$ CI 0.92 to $1.33, P=0.29$ ), and neutropenia (RR $1.11,95 \%$ CI 0.88 to $1.40, P=0.36$ ). Conclusion. This meta-analysis showed that bevacizumab could increase ORR, OS, and prolonged PFS for treatment of NSqNSCLC patients. However, no significant improvement in DCR was observed and bevacizumab could increase the rate of hypertension and hemorrhagic events. Bevacizumab was an acceptable option for NSqNSCLC patients. This trial is registered with PROSPERO registration number: CRD42021226790.
\end{abstract}

\section{Introduction}

As the leading cause of cancer death worldwide, lung cancer accounts for $18.4 \%$ of the total cancer deaths [1]. Non-smallcell lung cancer (NSCLC) accounts for approximately $80-$ $85 \%$ of all lung cancer cases and usually allocated to advanced stage at their first diagnosis [2]. The NCCN guideline suggested that systemic palliative chemotherapy and/or radiotherapy remained the standard care for these locally advanced or metastatic NSCLC patients [3].

To inhibit angiogenesis is another treatment option, because tumor angiogenesis is critical for the process of primary tumor growth, proliferation, differentiation, and metastasis and has been identified as an important therapeutic target for tumor in recent decades $[4,5]$. Antiangiogenic therapy has been used for cancer treatment, which inhibits the delivery of oxygen and nutrients to cancer cells [6]. As a key mediator of angiogenesis, vascular endothelial growth factor (VEGF) and its receptors are considered to be the pivotal pathway in angiogenesis-related molecular mechanisms which have been well studied $[7,8]$.

Bevacizumab is a VEGF monoclonal antibody, which inhibits angiogenesis to suppress tumor growth by restricting oxygen and nutrient supply to tumors [9]. Increasing 
numbers of clinical trials have been conducted with bevacizumab for the treatment of patients with advanced NSCLC since it was approved. Previous meta-analysis found that bevacizumab used in combination with paclitaxel and carboplatin did increase objective response rate (ORR), overall survival (OS), and prolonged progression-free survival (PFS) compared with paclitaxel and carboplatin for NSCLC [10-14]. However, the important outcome disease control rate (DCR) was not analyzed and only five RCTs were included. Whether bevacizumab containing could improve ORR, DCR, and OS and increase adverse reactions for treatment of nonsquamous non-small-cell lung cancer (NSqNSCLC) patients is still controversial. In consideration of these controversial results, we carried out this meta-analysis. Our meta-analysis includes 10 randomized controlled trials (RCTs) and identifies the precise effect of bevacizumab containing for NSqNSCLC patients on outcomes of ORR, DCR, OS, PFS, and treatmentrelated adverse event compared with bevacizumab free.

\section{Method}

2.1. Protocol and Registration. This meta-analysis was performed according to the PRISMA (Preferred Reporting Items for Systematic Reviews and Meta-Analyses) recommendations. This study was not a human or animal experiment; thus, ethical approval was not necessary. A protocol for this meta-analysis has been registered on PROSPERO (http://www.crd.york.ac.uk/prospero), and the registration number is CRD42021226790.

2.2. Search Strategy. Databases including PubMed, EMBASE, and the Cochrane Central Register of Controlled Trials were searched with a combination of the terms "non-small-cell lung cancer (NSCLC) or nonsquamous non-small-cell lung cancer (NSqNSCLC)" and "angiogenesis inhibitors or bevacizumab" within the restriction limit of "randomized controlled trial (RCT)." In addition, reference lists of the included studies were manually checked for potentially eligible studies and Google Scholar search engines were used to find additional references. The last search was performed on December 8, 2020, without any restriction to language of publication.

2.3. Inclusion and Exclusion Criteria. Inclusion criteria are as follows: (1) research types: RCTs publicly published at home and abroad; (2) research objects: adult patients with confirmed locally advanced or metastatic NSqNSCLC; and (3) intervention measures: the experimental group using bevacizumab plus standard chemotherapy regimen and the control group using standard chemotherapy regimen alone. Exclusion criteria are as follows: exclude articles that do not meet the inclusion criteria, cannot obtain the main indicators in the article, and have not received a response through contacting the author, and republished articles.

2.4. Quality Assessment and Data Extraction. The Cochrane risk of bias tool was used to evaluate the quality of each study by two reviewers, and the following 7 categories were assessed: random sequence generation, allocation concealment, blinding of participants and personnel, blinding of the outcome assessment, incomplete outcome data, selective outcome reporting, and other biases [15]. The overall methodologic quality of each included study was assessed as "low risk of bias," "high risk of bias," or "unclear risk of bias." A third reviewer would be invited if there were any dispute.

Two authors independently completed the data extraction. The extracted general data included author, year, and country of publication and sample size. The primary endpoint was OS, and the secondary end-points contained ORR, DCR, PFS, grade 3-5 of treatment-related adverse event (TRAE3-5), hypertension neutropenia, thrombocytopenia, anemia, and hemorrhagic events.

2.5. Statistical Analysis. Outcomes were estimated by calculating the pooled risk ratio (RR) (95\% confidence intervals [CIs]) for ORR, DCR, and TRAE by RevMan software (version 5.1; Cochrane Collaboration, Copenhagen, Denmark), and hazard ratio was pooled for survival outcomes (OS and PFS) by STATA version 12.0 (StataCorp, College Station, TX). A $P<0.05$ was considered statistically significant. Heterogeneity was assessed by visual inspection of the forest plot combined with the results of the test for heterogeneity and the $I^{2}$ test. Fixed-effects model would be employed for outcomes with low heterogeneity $\left(I^{2}<50 \%\right)$. Otherwise, the random-effects model of DerSimonian and Laird [16] would be selected. Sensitivity analysis would be conducted by omission of each single study to evaluate stability of the results if heterogeneous studies existed.

\section{Results}

3.1. Search Results and Characteristics of Included Studies. 551 potential articles were initially identified through database searches on 8 December 2020. Two hundred and thirty-three studies were considered potentially eligible for further assessment after duplicates were removed. Finally, 10 RCTs [17-26] that involved a total of 3134 patients published between 2006 and 2020 met the inclusion criteria and were included in this meta-analysis after a full-text review. Figure 1 shows the literature selection process. Table 1 summarizes the details of both the included studies and agents.

3.2. Risk of Bias. All included RCTs were assessed by two authors independently according to Cochrane risk of bias tool. Detailed information can be found in Figure 2.

\subsection{Outcomes of the Bevacizumab-Containing Group versus the Bevacizumab-Free Group}

3.3.1. Efficacy Profile. Compared to the bevacizumab-free group, the bevacizumab-containing group was associated with significantly superior ORR (RR 1.63 , 95\% CI 1.24 to 2.14, $P<0.001$; Figure 3), OS (HR 0.90, 95\% CI 0.82 to $0.99, z=21.45, P<0.001$; Figure 4 ), and longer PFS (HR $0.68,95 \%$ CI 0.62 to $0.74, z=22.50, P<0.001$; Figure 5). However, no significant improvement in DCR (RR 1.13, $95 \%$ CI 0.99 to $1.30, P=0.08$; Figure 6 ) was observed.

3.3.2. Safety Profile. For TRAE3-5, thrombocytopenia, and neutropenia outcomes, no significant difference was 


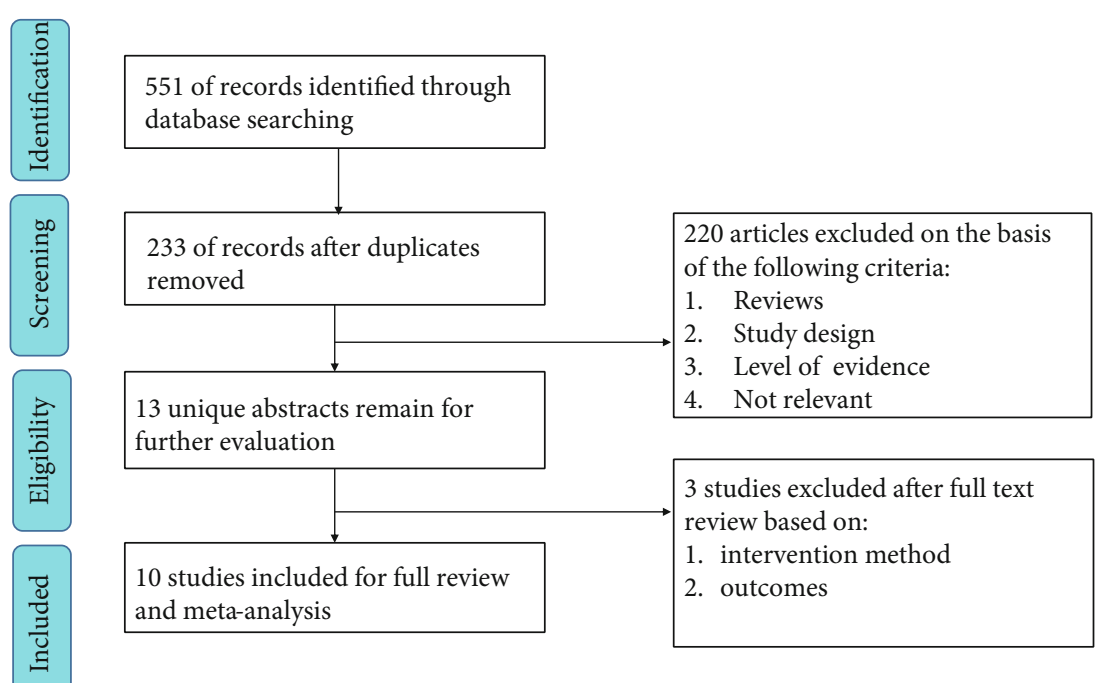

FIGURE 1: Flow diagram shows the process of literature selection.

TABle 1: Characteristics of included studies.

\begin{tabular}{|c|c|c|c|c|c|}
\hline \multirow{2}{*}{ Studies } & \multirow{2}{*}{ Year } & \multicolumn{2}{|c|}{ Intervention } & \multirow{2}{*}{$\begin{array}{l}\text { Number (case/ } \\
\text { control) }\end{array}$} & \multirow{2}{*}{$\begin{array}{l}\text { Type of } \\
\text { study }\end{array}$} \\
\hline & & Experimental group & Control group & & \\
\hline Cortot et al. & 2020 & Bevacizumab+paclitaxel & Docetaxel & $111 / 55$ & RCT \\
\hline Kitagawa et al. & 2019 & Bevacizumab+gefitinib & Gefitinib & $6 / 10$ & RCT \\
\hline Fukuda et al. & 2019 & Bevacizumab+pemetrexed & Pemetrexed & $20 / 20$ & RCT \\
\hline Saito et al. & 2019 & Bevacizumab+erlotinib & Erlotinib & $114 / 114$ & RCT \\
\hline $\begin{array}{l}\text { Karayama } \\
\text { et al. }\end{array}$ & 2016 & Bevacizumab+pemetrexed & Pemetrexed & $55 / 55$ & RCT \\
\hline Seto et al. & 2014 & Bevacizumab+erlotinib & Erlotinib & $77 / 77$ & RCT \\
\hline Niho et al. & 2012 & Bevacizumab+carboplatin+paclitaxel & Carboplatin+paclitaxel & $121 / 59$ & RCT \\
\hline Reck et al. & 2010 & $\begin{array}{c}\text { Bevacizumab } 7.5 \mathrm{mg} / \mathrm{kg}+\text { cisplatin } \\
\text { +gemcitabine }\end{array}$ & $\begin{array}{l}\text { Placebo+cisplatin } \\
\text { +gemcitabine }\end{array}$ & $345 / 347$ & RCT \\
\hline Reck et al. & 2009 & $\begin{array}{c}\text { Bevacizumab } 15 \mathrm{mg} / \mathrm{kg}+\text { cisplatin } \\
\text { +gemcitabine }\end{array}$ & $\begin{array}{l}\text { Placebo+cisplatin } \\
\text { +gemcitabine }\end{array}$ & $351 / 347$ & RCT \\
\hline Sandler et al. & 2006 & Bevacizumab+paclitaxel+carboplatin & Paclitaxel+carboplatin & $417 / 433$ & RCT \\
\hline
\end{tabular}

RCT: randomized controlled trial.

observed between the bevacizumab-containing group and bevacizumab-free group (RR $1.23,95 \%$ CI 0.99 to $1.53, P$ $=0.06 ; \mathrm{RR} 1.11,95 \% \mathrm{CI} 0.92$ to $1.33, P=0.29$; and RR $1.11,95 \%$ CI 0.88 to $1.40, P=0.36$ Figures $7-9)$. The bevacizumab-containing group showed higher rate of hypertension and hemorrhagic events than the bevacizumab-free group (RR 6.91, 95\% CI 4.62 to $10.35, P<0.00001$ and RR 3.07, $95 \%$ CI 1.78 to 5.30, $P<0.0001$; Figures 10 and 11 ). The bevacizumab-containing group showed lower rate of anemia than the bevacizumab-free group (RR 0.72, 95\% CI 0.55 to $0.96, P=0.02$; Figure 12 ).

3.3.3. Sensitivity Analysis. Sensitivity analysis indicated that omitting any single study did not significantly affect the pooled RR for ORR (Table 2). For DCR, omitting Saito (2019) showed that $I^{2}$ was decreased to $45 \%$ and significant difference was observed (RR 1.18, 95\% CI 1.05 to
1.32, $P<0.01$; Table 3). For TRAE3-5, omitting Cortot (2020) showed that $I^{2}$ was $93 \%$ and significant difference was observed (RR 1.32, 95\% CI 1.04 to $1.68, P<0.05$; Table 4). For neutropenia, omitting Cortot (2020) showed that $I^{2}$ was decreased to $48 \%$ and significant difference was observed (RR 1.23, 95\% CI 1.05 to $1.44, P=0.01$; Table 5).

\section{Discussion}

Interference with VEGFR functions has been an alternative approach for the treatment of NSCLC [27]. Bevacizumab, a novel targeted therapeutic, differs in their modes of action and tolerability profiles from those of cytotoxic agents and can be combined with traditional chemotherapy to offer greater clinical benefits [25]. It has been approved for use in combination with the standard platinum-based chemotherapy or as a maintenance therapy after chemotherapy 


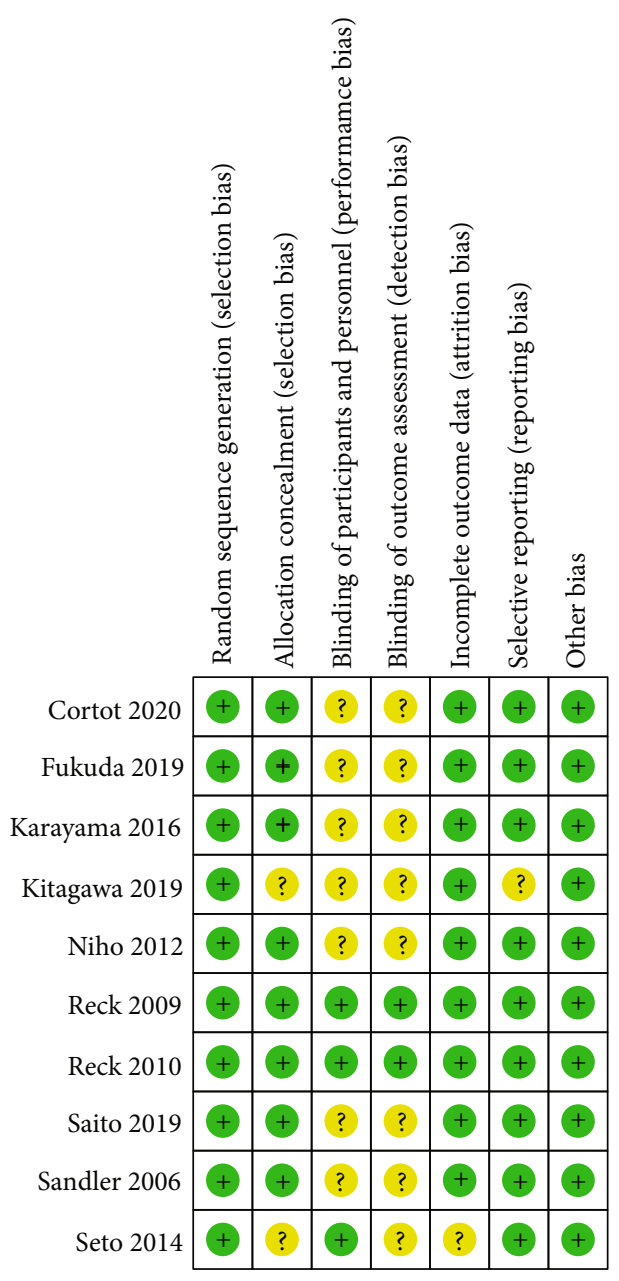

FIgURE 2: The methodological quality of the RCTs.

\begin{tabular}{|c|c|c|c|c|c|c|c|c|c|c|c|c|}
\hline \multirow{2}{*}{$\frac{\text { Study or subgroup }}{\text { Cortot } 2020}$} & \multicolumn{2}{|c|}{ Experimental } & \multicolumn{2}{|c|}{ Control } & Weight & $\begin{array}{c}\text { Risk ratio } \\
\mathrm{M}-\mathrm{H} \text {, random, } 95 \% \mathrm{Cl}\end{array}$ & \multicolumn{6}{|c|}{$\begin{array}{c}\text { Risk ratio } \\
\text { M-H, random, } 95 \% \mathrm{Cl}\end{array}$} \\
\hline & 25 & 111 & 3 & 55 & $4.2 \%$ & $4.13[1.30,13.08]$ & & & & & & \\
\hline Fukuda 2019 & 11 & 20 & 3 & 20 & $4.4 \%$ & $3.67[1.20,11.19]$ & & & & & & \\
\hline Kitagawa 2019 & 3 & 6 & 4 & 9 & $4.6 \%$ & $1.13[0.38,3.32]$ & & & & & & \\
\hline Niho 2012 & 71 & 117 & 18 & 58 & $12.2 \%$ & $1.96[1.30,2.95]$ & & & & & & \\
\hline Reck 2009 & 100 & 329 & 66 & 327 & $14.5 \%$ & $1.51[1.15,1.97]$ & & & & 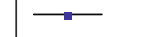 & & \\
\hline Reck 2010 & 113 & 330 & 66 & 327 & $14.6 \%$ & $1.70[1.31,2.21]$ & & & & $\longrightarrow$ & & \\
\hline Saito 2019 & 81 & 114 & 74 & 114 & $15.8 \%$ & $1.09[1.92,1.31]$ & & & & & & \\
\hline Sandler 2006 & 133 & 381 & 59 & 392 & $14.5 \%$ & $2.32[1.77,3.05]$ & & & & - & & \\
\hline Seto 2014 & 52 & 77 & 49 & 77 & $15.1 \%$ & $1.06[0.84,1.33]$ & & & & & & \\
\hline Total $(95 \% \mathrm{Cl})$ & & 1485 & & 1379 & $100.0 \%$ & $1.63[1.24,2.14)$ & & & & & & \\
\hline \multirow{3}{*}{\multicolumn{7}{|c|}{$\begin{array}{l}\text { Total events } \\
\text { Heterogeneity: } \mathrm{Tau}^{2}=0.11 ; \mathrm{Chi}^{2}=43.51, \mathrm{df} \\
\text { Test for overall effect: } Z=3.50(P<0.0005)\end{array}$}} & & & & & & \\
\hline & & & & & & & 0.1 & 0.2 & 0.5 & 1 & 5 & 10 \\
\hline & & & & & & & & Gavou & ntrol & Favours & peri & ntal \\
\hline
\end{tabular}

FIGURE 3: ORR.

during the treatment of NSCLC patients without driver mutations $[26,28]$. However, little information was reported on DCR and TRAE of NSqNSCLC patients. Besides, there have been several novel studies published afterwards. Thus, it is necessary to update the results.

We conducted this meta-analysis with 10 RCTs included 3134 advanced NSqNSCLC patients to compare therapeutic efficacy and adverse reactions of bevacizumab containing and bevacizumab free for NSqNSCLC patients. According to the current outcomes, treatment regimens containing bevacizumab had significant improvements for ORR, OS, and PFS outcomes, when compared with the treatment regimens without bevacizumab. However, significant outcome was not observed in DCR. It is indicated that significant 


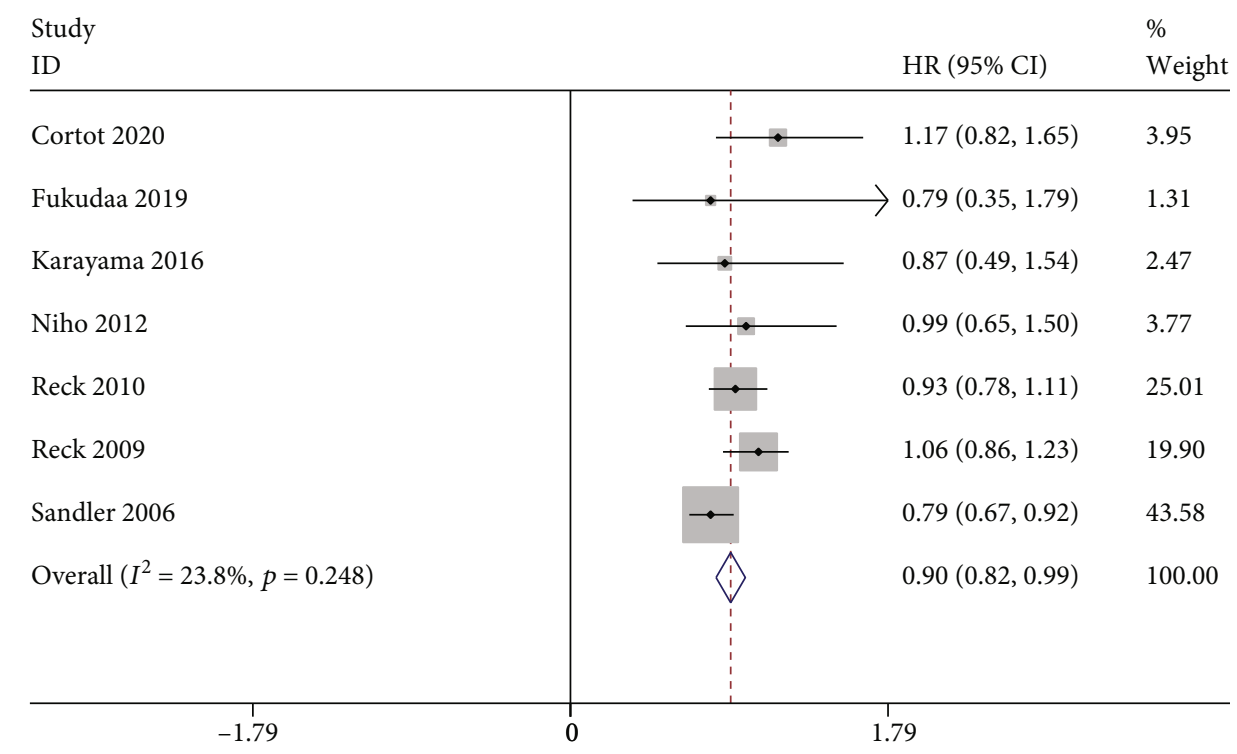

Figure 4: OS.

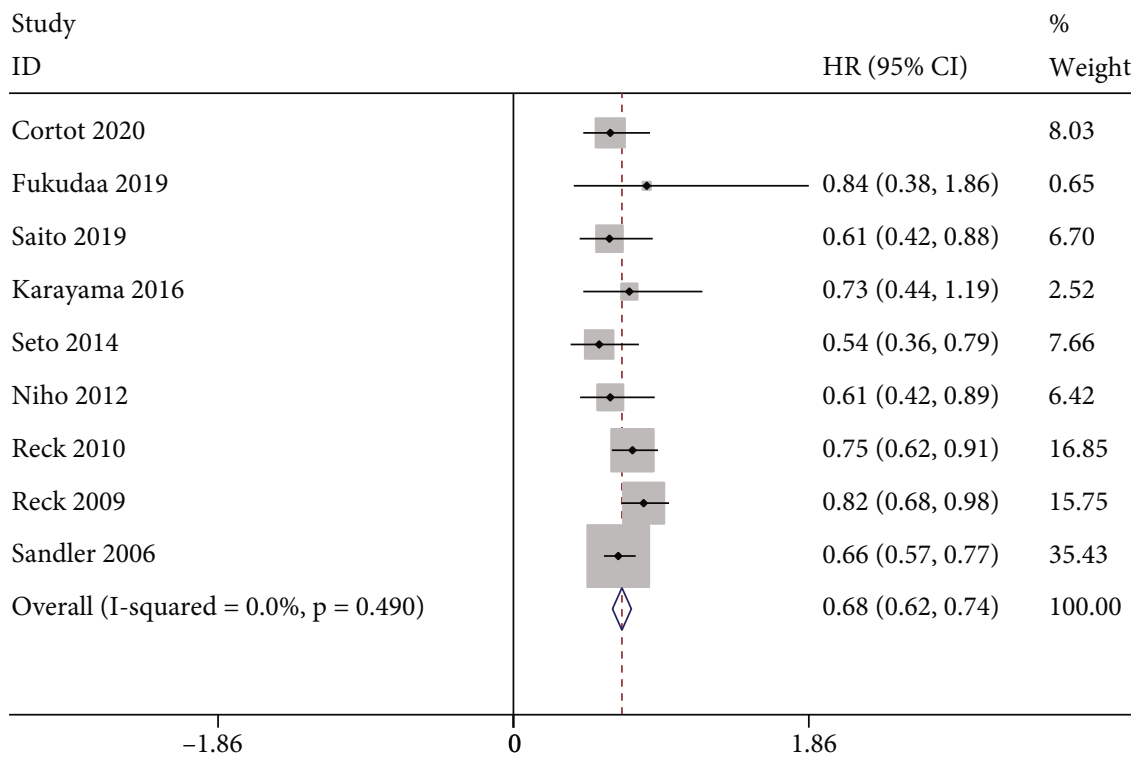

Figure 5: PFS.

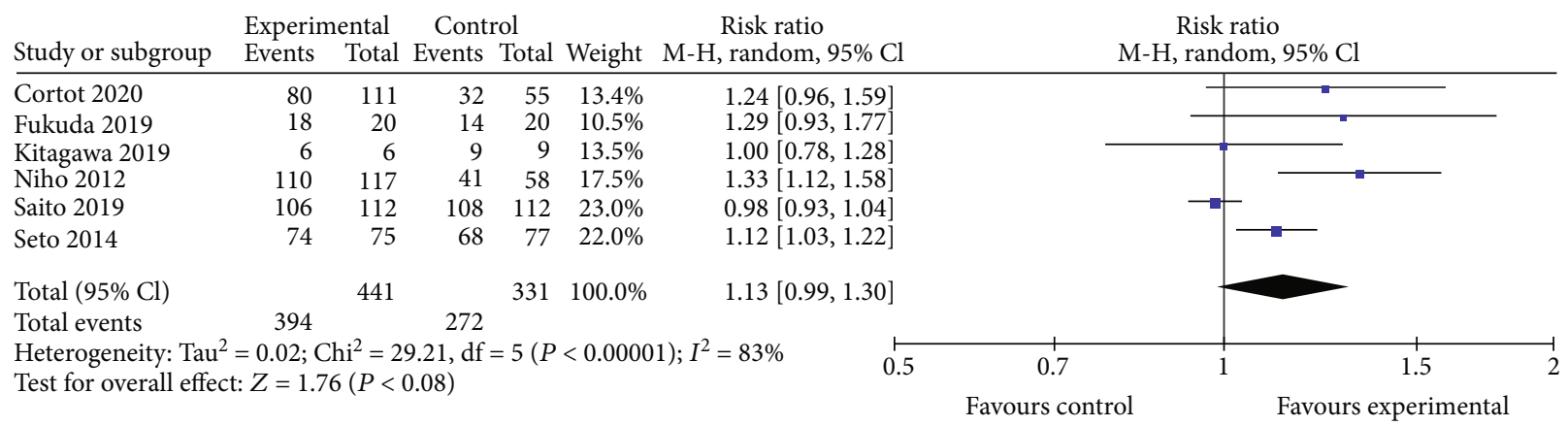

Figure 6: DCR. 


\begin{tabular}{|c|c|c|c|c|c|c|c|c|c|c|c|}
\hline Study or subgroup & \multicolumn{2}{|c|}{ Experimental } & \multicolumn{2}{|c|}{ Control } & Weight & $\begin{array}{c}\text { Risk ratio } \\
\mathrm{M}-\mathrm{H} \text {, random, } 95 \% \mathrm{Cl}\end{array}$ & \multicolumn{5}{|c|}{ Risk ratio } \\
\hline Cortot 2020 & 50 & 109 & 30 & 55 & $15.7 \%$ & $0.84[0.61,1.15]$ & & & & & \\
\hline Reck 2009 & 265 & 329 & 246 & 327 & $23.0 \%$ & $1.07[0.99,1.16]$ & & & & & \\
\hline Reck 2010 & 252 & 330 & 246 & 327 & $22.9 \%$ & $1.02[0.93,1.11]$ & & & & & \\
\hline Saito 2019 & 98 & 112 & 53 & 114 & $19.4 \%$ & $1.88[1.53,2.32]$ & & & & & \\
\hline Seto 2014 & 68 & 75 & 41 & 74 & $19.1 \%$ & $1.64[1.32,2.03]$ & & & & & \\
\hline Total $(95 \% \mathrm{Cl})$ & & 955 & & 897 & $100.0 \%$ & $1.23[0.99,1.53]$ & & & & & \\
\hline \multirow{3}{*}{\multicolumn{7}{|c|}{$\begin{array}{l}\text { Total events } \quad 733 \quad 616 \\
\text { Heterogeneity: } \mathrm{Tau}^{2}=0.05 ; \mathrm{Chi}^{2}=45.48, \mathrm{df}=4(P<0.00001) ; I^{2}=91 \% \\
\text { Test for overall effect: } Z=1.91(P<0.06)\end{array}$}} & & & & & \\
\hline & & & & & & & 0.5 & 0.7 & 1 & 1.5 & 2 \\
\hline & & & & & & & Favo & ont & & ou & \\
\hline
\end{tabular}

FIGURE 7: TRAE3-5.

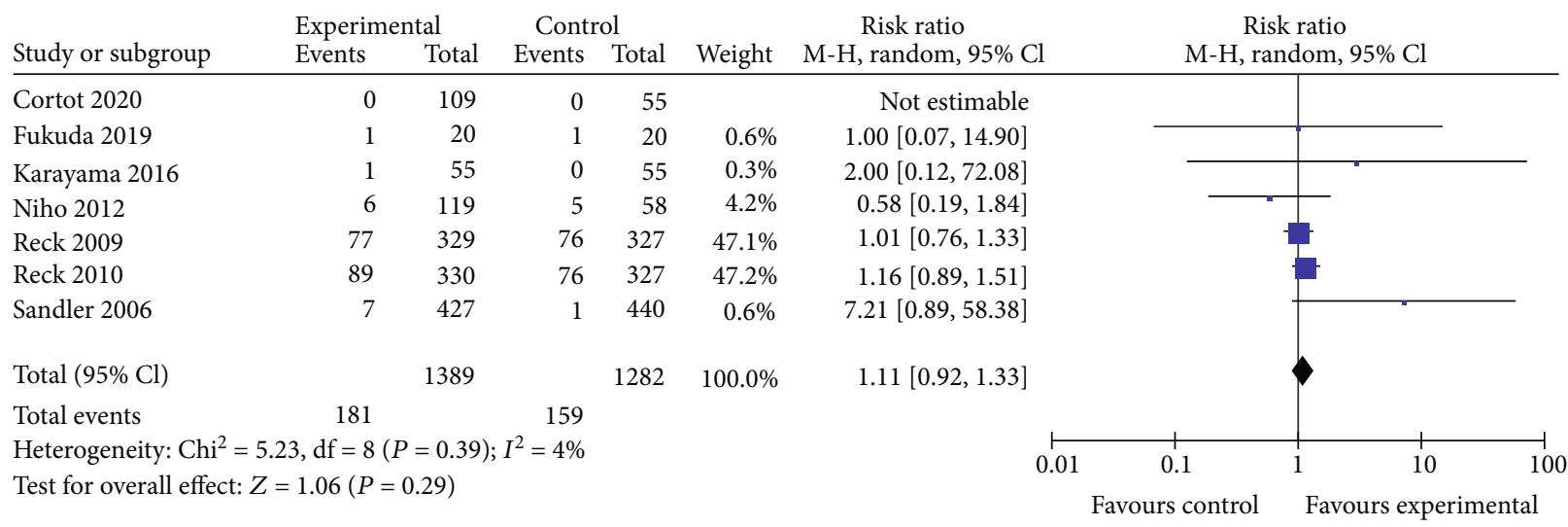

FIgURE 8: Thrombocytopenia.

\begin{tabular}{|c|c|c|c|c|c|c|c|c|c|}
\hline \multirow{2}{*}{$\frac{\text { Study or subgroup }}{\text { Cortot } 2020}$} & \multicolumn{2}{|c|}{ Experimental } & \multicolumn{2}{|c|}{ Control } & Weight & $\begin{array}{c}\text { Risk ratio } \\
\mathrm{M}-\mathrm{H}, \text { random, } 95 \% \mathrm{Cl}\end{array}$ & \multicolumn{2}{|c|}{$\begin{array}{c}\text { Risk ratio } \\
\mathrm{M}-\mathrm{H}, \text { random, } 95 \% \mathrm{Cl}\end{array}$} & \\
\hline & 21 & 109 & 25 & 55 & $11.8 \%$ & $0.42[0.26,0.69]$ & -- & & \\
\hline Fukuda 2019 & 11 & 20 & 4 & 20 & $4.7 \%$ & $2.75[1.05,7.20]$ & & & \\
\hline Kitagawa 2019 & 3 & 55 & 3 & 55 & $2.0 \%$ & $1.00[0.21,4.74]$ & & & \\
\hline Niho 2012 & 108 & 119 & 49 & 58 & $22.5 \%$ & $1.07[0.95,1.22]$ & & 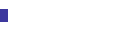 & \\
\hline Reck 2009 & 117 & 329 & 104 & 327 & $19.9 \%$ & $1.12[0.90,1.39]$ & & 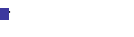 & \\
\hline Reck 2010 & 132 & 330 & 104 & 327 & $20.2 \%$ & $1.26[1.02,1.55]$ & & $=$ & \\
\hline Saito 2019 & 1 & 112 & 1 & 114 & $0.7 \%$ & $1.02[0.06,16.07]$ & & & \\
\hline Sandler 2006 & 109 & 427 & 74 & 440 & $18.3 \%$ & $1.52[1.17,1.98]$ & & $=$ & \\
\hline Total $(95 \% \mathrm{Cl})$ & & 1501 & & 1396 & $100.0 \%$ & $1.11[0.88,1.40]$ & & & \\
\hline Total events & 502 & & 364 & & & & & & \\
\hline \multicolumn{6}{|c|}{ Heterogeneity: $\mathrm{Tau}^{2}=0.06 ; \mathrm{Chi}^{2}=26.71, \mathrm{df}=7(P=0.0004) ; I^{2}=74 \%$} & 0.001 & 0.1 & 10 & 1000 \\
\hline
\end{tabular}

FIgURE 9: Neutropenia.

improvements of ORR and PFS could translate into overall survival benefits. A previous meta-analysis reported similar results regarding efficacy profile [10]. However, the important outcome disease control rate (DCR) was not analyzed and only five RCTs were included. Five RCTs including 1852 patients, 7 RCTs including 2671 patients, and 8 RCTs including 2897 patients indicated that the bevacizumabcontaining group and bevacizumab-free group had a similar rate of TRAE3-5, thrombocytopenia, and neutropenia. All
10 RCTs reported hypertension and 7 RCTs reported hemorrhagic events, and the bevacizumab-containing group showed a higher rate of hypertension and hemorrhagic events compared to the bevacizumab-free group. Seven RCTs reported anemia and the bevacizumab-containing group showed a lower rate of anemia compared to the bevacizumab-free group.

Though more RCTs were included in this meta-analysis, improvement was not observed with respect to DCR in the 


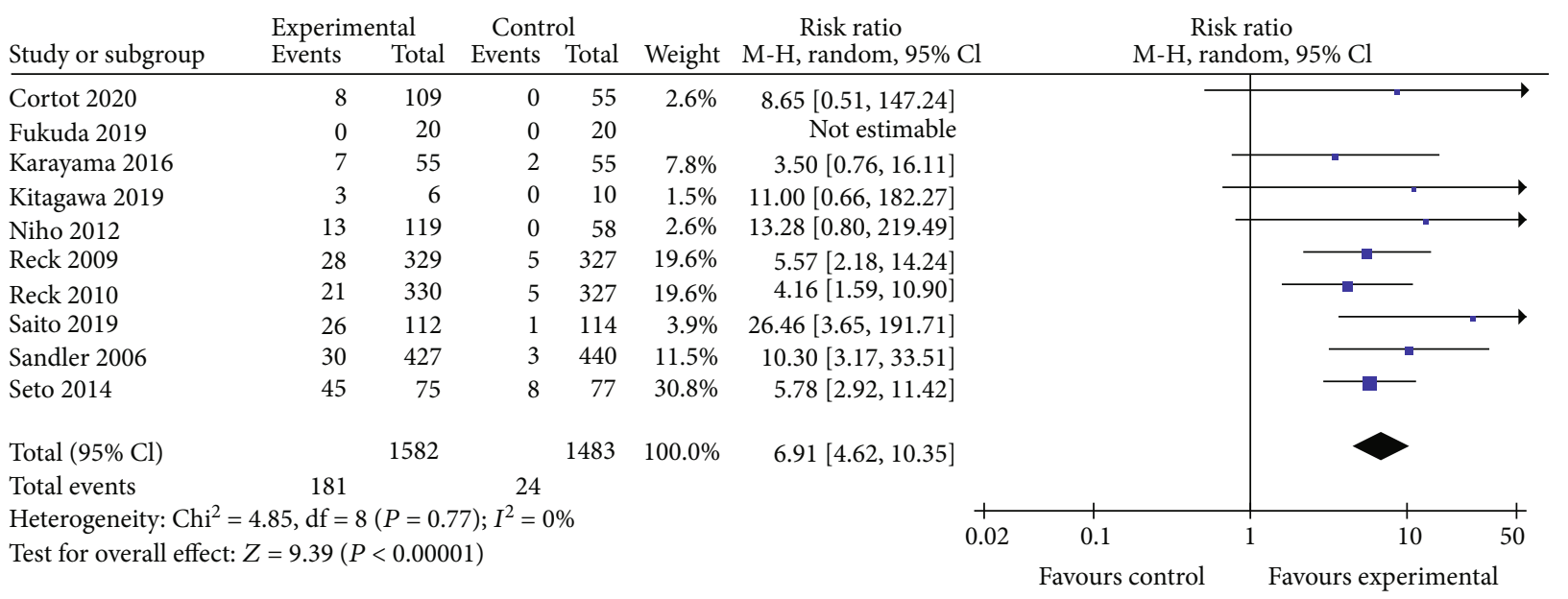

FIGURE 10: Hypertension.

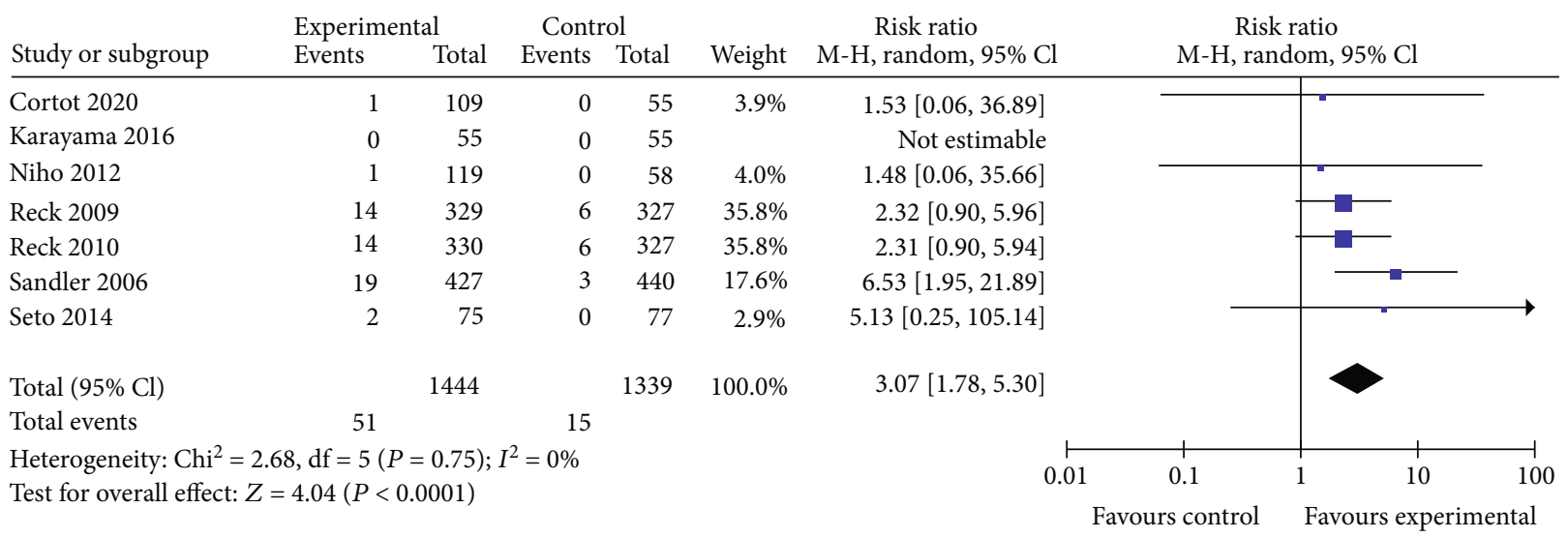

FIGURE 11: Hemorrhagic event.

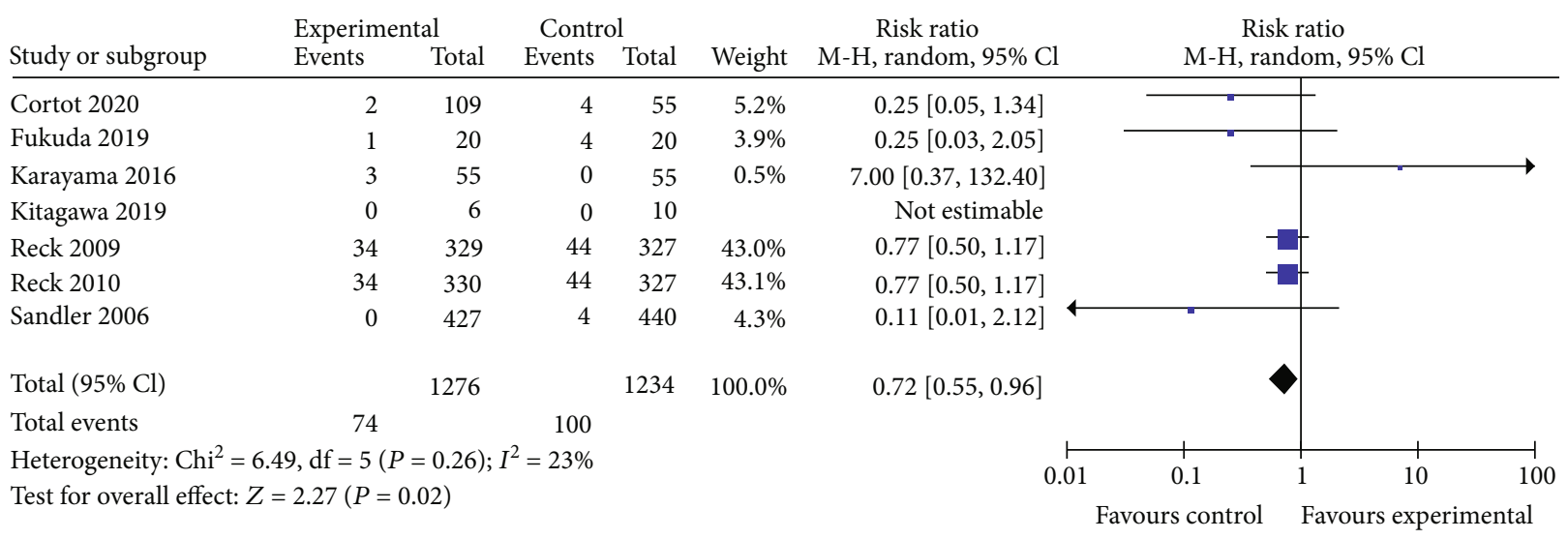

Figure 12: Anemia.

bevacizumab-containing group. A possible reason is that Saito (2019) affected the result, because sensitivity analysis indicated that omitting Saito (2019) showed that $I^{2}$ was decreased to $45 \%$ and significant difference was observed for DCR. Other possible reasons include that bevacizumab may not improve
DCR or limited number of RCTs limits the positive result. More multicenter, large-sample RCTs or even real-world studies comparing bevacizumab for NSqNSCLC patients are urged to validate the DCR as DCR was considered as one of the important outcomes for cancer patients. 
TABLE 2: Sensitivity analyses based on various exclusion criteria for ORR.

\begin{tabular}{lcccccccc}
\hline Excluded trial & $\begin{array}{c}\text { No. of } \\
\text { trials }\end{array}$ & $\begin{array}{c}\text { No. of } \\
\text { patients }\end{array}$ & $\begin{array}{c}\text { Experimental } \\
\text { group }\end{array}$ & $\begin{array}{c}\text { Control } \\
\text { group }\end{array}$ & RR (95\% CI) & $\begin{array}{c}P \text { value for } \\
\text { RR }\end{array}$ & $\begin{array}{c}I^{2} \\
(\%)\end{array}$ & $\begin{array}{c}P \text { value for } \\
\text { heterogeneity }\end{array}$ \\
\hline Cortot (2020) & 8 & 2698 & 1374 & 1324 & $1.56[1.19,2.04]$ & $<0.01$ & 82 & $<0.01$ \\
Fukuda (2019) & 8 & 2824 & 1465 & 1359 & $1.56[1.19,2.06]$ & $<0.01$ & 83 & $<0.01$ \\
Kitagawa & 8 & 2849 & 1479 & 1370 & $1.66[1.25,2.20]$ & $<0.01$ & 84 & $<0.01$ \\
$($ 2019) & 8 & 2689 & 1368 & 1321 & $1.59[1.18,2.14]$ & $<0.01$ & 83 & $<0.01$ \\
Niho (2012) & 8 & 2208 & 1156 & 1052 & $1.67[1.21,2.31]$ & $<0.01$ & 84 & $<0.01$ \\
Reck (2009) & 8 & 1155 & 1052 & $1.63[1.19,2.24]$ & $<0.01$ & 83 & $<0.01$ \\
Reck (2010) & 8 & 2207 & 1371 & 1265 & $1.74[1.31,2.31]$ & $<0.01$ & 75 & $<0.01$ \\
Saito (2019) & 8 & 2636 & 1104 & 987 & $1.50[1.16,1.93]$ & $<0.01$ & 74 & $<0.01$ \\
Sandler (2006) & 8 & 2091 & 1408 & 1302 & $1.75[1.31,2.36]$ & $<0.01$ & 79 & $<0.01$ \\
Seto (2014) & 8 & 2710 & & & & &
\end{tabular}

TABLe 3: Sensitivity analyses based on various exclusion criteria for DCR.

\begin{tabular}{lcccccccc}
\hline Excluded trial & $\begin{array}{c}\text { No. of } \\
\text { trials }\end{array}$ & $\begin{array}{c}\text { No. of } \\
\text { patients }\end{array}$ & $\begin{array}{c}\text { Experimental } \\
\text { group }\end{array}$ & $\begin{array}{c}\text { Control } \\
\text { group }\end{array}$ & RR (95\% CI) & $\begin{array}{c}P \text { value for } \\
\text { RR }\end{array}$ & $\begin{array}{c}I^{2} \\
(\%)\end{array}$ & $\begin{array}{c}P \text { value for } \\
\text { heterogeneity }\end{array}$ \\
\hline Cortot (2020) & 5 & 554 & 314 & 240 & $1.12[0.97,1.29]$ & $>0.05$ & 83 & $<0.01$ \\
Fukuda (2019) & 5 & 732 & 421 & 311 & $1.12[0.97,1.29]$ & $>0.05$ & 85 & $<0.01$ \\
Kitagawa (2019) & 5 & 757 & 435 & 322 & $1.16[0.99,1.35]$ & $>0.05$ & 86 & $<0.01$ \\
Niho (2012) & 5 & 597 & 324 & 273 & $1.09[0.96,1.23]$ & $>0.05$ & 73 & $<0.01$ \\
Saito (2019) & 5 & 548 & 329 & 219 & $1.18[1.05,1.32]$ & $<0.01$ & 45 & $<0.01$ \\
Seto (2014) & 5 & 620 & 366 & 254 & $1.15[0.92,1.43]$ & $>0.05$ & 87 & $<0.01$ \\
\hline
\end{tabular}

TABLE 4: Sensitivity analyses based on various exclusion criteria for TRAE3-5.

\begin{tabular}{lcccccccc}
\hline $\begin{array}{l}\text { Excluded } \\
\text { trial }\end{array}$ & $\begin{array}{c}\text { No. of } \\
\text { trials }\end{array}$ & $\begin{array}{c}\text { No. of } \\
\text { patients }\end{array}$ & $\begin{array}{c}\text { Experimental } \\
\text { group }\end{array}$ & $\begin{array}{c}\text { Control } \\
\text { group }\end{array}$ & RR (95\% CI) & $\begin{array}{c}P \text { value for } \\
\text { RR }\end{array}$ & $\begin{array}{c}I^{2} \\
(\%)\end{array}$ & $\begin{array}{c}P \text { value for } \\
\text { heterogeneity }\end{array}$ \\
\hline $\begin{array}{l}\text { Cortot } \\
(2020)\end{array}$ & 4 & 554 & 846 & 842 & $1.32[1.04,1.68]$ & $<0.05$ & 93 & $<0.01$ \\
Reck (2009) & 4 & 732 & 626 & 570 & $1.28[0.89,1.84]$ & $>0.05$ & 93 & $<0.01$ \\
Reck (2010) & 4 & 757 & 625 & 570 & $1.30[0.93,1.82]$ & $>0.05$ & 92 & $<0.01$ \\
Saito (2019) & 4 & 597 & 635 & 563 & $1.11[0.94,1.32]$ & $>0.05$ & 84 & $<0.01$ \\
Seto (2014) & 4 & 548 & 880 & 823 & $1.15[0.93,1.43]$ & $>0.05$ & 91 & $<0.01$ \\
\hline
\end{tabular}

TABLE 5: Sensitivity analyses based on various exclusion criteria for neutropenia.

\begin{tabular}{lcccccccc}
\hline Excluded trial & $\begin{array}{c}\text { No. of } \\
\text { trials }\end{array}$ & $\begin{array}{c}\text { No. of } \\
\text { patients }\end{array}$ & $\begin{array}{c}\text { Experimental } \\
\text { group }\end{array}$ & $\begin{array}{c}\text { Control } \\
\text { group }\end{array}$ & RR (95\% CI) & $\begin{array}{c}P \text { value for } \\
\text { RR }\end{array}$ & $\begin{array}{c}I^{2} \\
(\%)\end{array}$ & $\begin{array}{c}P \text { value for } \\
\text { heterogeneity }\end{array}$ \\
\hline Cortot (2020) & 7 & 2733 & 1392 & 1341 & $1.25[1.12,1.39]$ & $<0.0001$ & 48 & 0.07 \\
Fukuda (2019) & 7 & 2857 & 1481 & 1376 & $1.07[0.85,1.34]$ & 0.56 & 74 & 0.0008 \\
Kitagawa (2019) & 7 & 2789 & 1446 & 1341 & $1.12[0.88,1.42]$ & 0.37 & 78 & 0.0002 \\
Niho (2012) & 7 & 2720 & 1382 & 1338 & $1.12[0.81,1.55]$ & 0.50 & 76 & 0.0004 \\
Reck (2009) & 7 & 2241 & 1172 & 1069 & $1.11[0.82,1.50]$ & 0.51 & 78 & 0.0001 \\
Reck (2010) & 7 & 2240 & 1171 & 1069 & $1.08[0.80,1.45]$ & 0.62 & 76 & 0.0003 \\
Saito (2019) & 7 & 2671 & 1389 & 1282 & $1.11[0.88,1.41]$ & 0.37 & 78 & 0.0002 \\
Sandler (2006) & 7 & 2030 & 1074 & 956 & $1.04[0.81,1.33]$ & 0.76 & 71 & 0.002 \\
\hline
\end{tabular}




\section{Limitations of This Study}

First, we did not perform subgroup analysis as subgroup analysis will result in limited articles available. Second, various chemotherapeutic regimens and patterns are involved in different RCTs. This may lead to a certain degree of heterogeneity and significant heterogeneities. Third, we cannot extract more data to complete an in-depth analysis of DCR and more high-quality trials are warranted to support the survival benefit of bevacizumab.

\section{Conclusions}

Our meta-analysis showed that treatment containing bevacizumab was an option for patients with NSqNSCLC and patients with acceptable efficacy. Bevacizumab was superior to those without it in terms of ORR, OS, and PFS in patients with NSqNSCLC and no significant TRAE3-5 was observed.

\section{Data Availability}

This is a meta-analysis and all relevant data have been displayed in the manuscript.

\section{Conflicts of Interest}

The authors declare that they have no conflicts of interest.

\section{Authors' Contributions}

Yue Zhou and Mei He contributed equally to this work. Ming Yang made a contribution to designing the study and providing critical revisions to this article. Yue Zhou and Mei He were responsible to collect the data and write the article.

\section{Acknowledgments}

The authors thank all the participants of the studies included in the present study.

\section{References}

[1] F. Bray, J. Ferlay, I. Soerjomataram, R. L. Siegel, L. A. Torre, and A. Jemal, "Global cancer statistics 2018: GLOBOCAN estimates of incidence and mortality worldwide for 36 cancers in 185 countries," CA: a Cancer Journal for Clinicians, vol. 68, no. 6, pp. 394-424, 2018.

[2] D. Iacono, R. Chiari, G. Metro et al., "Future options for $A L K$ -positive non-small cell lung cancer," Lung Cancer, vol. 87, no. 3, pp. 211-219, 2015.

[3] D. S. Ettinger, D. E. Wood, D. L. Aisner et al., "Non-small cell lung cancer, version 5.2017, NCCN clinical practice guidelines in oncology," Journal of the National Comprehensive Cancer Network, vol. 15, no. 4, pp. 504-535, 2017.

[4] T. A. Baudino, "Targeted cancer therapy: the next generation of cancer treatment," Current Drug Discovery Technologies, vol. 12, no. 1, pp. 3-20, 2015.

[5] D. Hanahan and R. A. Weinberg, "Hallmarks of cancer: the next generation," Cell, vol. 144, no. 5, pp. 646-674, 2011.
[6] M. Hong, H. Shi, N. Wang, H. Y. Tan, Q. Wang, and Y. Feng, "Dual effects of Chinese herbal medicines on angiogenesis in cancer and ischemic stroke treatments: role of HIF-1 network," Frontiers in Pharmacology, vol. 10, 2019.

[7] J. McLachlan and S. Banerjee, "Pazopanib in ovarian cancer," Expert Review of Anticancer Therapy, vol. 15, no. 9, pp. 9951005, 2015.

[8] C. Wang, L. Li, D. Fu et al., "Discovery of chalcone-modified estradiol analogs as antitumour agents that inhibit tumour angiogenesis and epithelial to mesenchymal transition," European Journal of Medicinal Chemistry, vol. 176, pp. 135-148, 2019.

[9] R. Midgley and D. Kerr, "Bevacizumab-current status and future directions," Annals of Oncology, vol. 16, no. 7, pp. 999-1004, 2005.

[10] S. Han, Y. Hong, T. Liu, N. Wu, and Z. Ye, “The efficacy and safety of paclitaxel and carboplatin with versus without bevacizumab in patients with non-small-cell lung cancer: a systematic review and meta-analysis," Oncotarget, vol. 9, no. 18, pp. 14619-14629, 2018.

[11] T. E. Botrel, O. Clark, L. Clark, L. Paladini, E. Faleiros, and B. Pegoretti, "Efficacy of bevacizumab (Bev) plus chemotherapy (CT) compared to CT alone in previously untreated locally advanced or metastatic non-small cell lung cancer (NSCLC): systematic review and meta-analysis," Lung Cancer, vol. 74, no. 1, pp. 89-97, 2011.

[12] A. B. Lima, L. T. Macedo, and A. D. Sasse, "Addition of bevacizumab to chemotherapy in advanced non-small cell lung cancer: a systematic review and meta-analysis," PLoS One, vol. 6, no. 8, p. e22681, 2011.

[13] V. Ranpura, S. Hapani, and S. Wu, "Treatment-related mortality with bevacizumab in cancer patients: a meta-analysis," JAMA, vol. 305, no. 5, pp. 487-494, 2011.

[14] J. C. Soria, A. Mauguen, M. Reck et al., "Systematic review and meta-analysis of randomised, phase II/III trials adding bevacizumab to platinum-based chemotherapy as first-line treatment in patients with advanced non-small-cell lung cancer," Annals of Oncology, vol. 24, no. 1, pp. 20-30, 2013.

[15] J. P. Higgins, D. G. Altman, P. C. Gotzsche et al., "The Cochrane Collaboration's tool for assessing risk of bias in randomised trials," BMJ, vol. 343, no. oct18 2, p. d5928, 2011.

[16] R. DerSimonian and N. Laird, "Meta-analysis in clinical trials," Controlled Clinical Trials, vol. 7, no. 3, pp. 177-188, 1986.

[17] A. B. Cortot, C. Audigier-Valette, O. Molinier et al., "Weekly paclitaxel plus bevacizumab versus docetaxel as second- or third-line treatment in advanced non-squamous non-smallcell lung cancer: results of the IFCT-1103 ULTIMATE study," European Journal of Cancer, vol. 131, pp. 27-36, 2020.

[18] H. Saito, T. Fukuhara, N. Furuya et al., "Erlotinib plus bevacizumab versus erlotinib alone in patients with EGFR -positive advanced non-squamous non-small-cell lung cancer (NEJ026): interim analysis of an open-label, randomised, multicentre, phase 3 trial," The Lancet Oncology, vol. 20, no. 5, pp. 625-635, 2019.

[19] C. Kitagawa, M. Mori, M. Ichiki et al., "Gefitinib plus Bevacizumabvs. gefitinib alone forEGFRMutant non-squamous non-small cell lung cancer," In Vivo, vol. 33, no. 2, pp. 477482, 2019.

[20] M. Fukuda, T. Kitazaki, D. Ogawara et al., "Randomized phase II study of pemetrexed or pemetrexed plus bevacizumab for elderly patients with previously untreated non-squamous 
non-small cell lung cancer: Results of the Lung Oncology Group in Kyushu (LOGIK1201)," Lung Cancer, vol. 132, pp. 1-8, 2019.

[21] M. Karayama, N. Inui, T. Fujisawa et al., "Maintenance therapy with pemetrexed and bevacizumab versus pemetrexed monotherapy after induction therapy with carboplatin, pemetrexed, and bevacizumab in patients with advanced nonsquamous non small cell lung cancer," European Journal of Cancer, vol. 58, pp. 30-37, 2016.

[22] T. Seto, T. Kato, M. Nishio et al., "Erlotinib alone or with bevacizumab as first-line therapy in patients with advanced non-squamous non-small-cell lung cancer harbouring EGFR mutations (JO25567): an open-label, randomised, multicentre, phase 2 study," The Lancet Oncology, vol. 15, no. 11, pp. 1236-1244, 2014.

[23] S. Niho, H. Kunitoh, H. Nokihara et al., "Randomized phase II study of first-line carboplatin-paclitaxel with or without bevacizumab in Japanese patients with advanced non-squamous non-small-cell lung cancer," Lung Cancer, vol. 76, no. 3, pp. 362-367, 2012.

[24] M. Reck, J. von Pawel, P. Zatloukal et al., "Overall survival with cisplatin-gemcitabine and bevacizumab or placebo as first-line therapy for nonsquamous non-small-cell lung cancer: results from a randomised phase III trial (AVAiL)," Annals of Oncology, vol. 21, no. 9, pp. 1804-1809, 2010.

[25] M. Reck, J. von Pawel, P. Zatloukal et al., "Phase III trial of cisplatin plus gemcitabine with either placebo or bevacizumab as first-line therapy for nonsquamous non-small-cell lung cancer: AVAil," Journal of Clinical Oncology, vol. 27, no. 8, pp. 12271234, 2009.

[26] A. Sandler, R. Gray, M. C. Perry et al., "Paclitaxel-carboplatin alone or with bevacizumab for non-small-cell lung cancer," The New England Journal of Medicine, vol. 355, no. 24, pp. 2542-2550, 2006.

[27] A. Spagnuolo, G. Palazzolo, C. Sementa, and C. Gridelli, "Vascular endothelial growth factor receptor tyrosine kinase inhibitors for the treatment of advanced non-small cell lung cancer," Expert Opinion on Pharmacotherapy, vol. 21, no. 4, pp. 491-506, 2020.

[28] R. Petrioli, E. Francini, A. I. Fiaschi et al., "Switch maintenance treatment with oral vinorelbine and bevacizumab after induction chemotherapy with cisplatin, gemcitabine and bevacizumab in patients with advanced non-squamous non-small cell lung cancer: a phase II study," Medical Oncology, vol. 32, no. $4,2015$. 